\title{
Oral Health Status of Chinese Paediatric and Adolescent Oncology Pa- tients with Chemotherapy in Hong Kong: a Pilot Study
}

\author{
Kung A.Y.H., Zhang S., Zheng L.W., Wong G.H.M. and Chu C.H.*
}

Faculty of Dentistry, the University of Hong Kong, Republic of China

\begin{abstract}
Aim: To study the oral health status of Chinese children and adolescents undergoing chemotherapy in Hong Kong.

Method: All Chinese children and adolescent oncology patients aged 18 or below attending the Children's Centre for Cancer and Blood Disease at a hospital for chemotherapy were invited and parental consent was sought before they were accepted into the study. The study comprised of 1) a parental questionnaire, 2) the collection of medical history and 3) a clinical examination for tooth decay (caries) and mucosal status.
\end{abstract}

Results: A total of 69 patients were invited, and they all participated in this study. Their mean age was $9.2 \pm 5.0$ and 44 $(64 \%)$ were males. Twenty-six patients $(38 \%)$ had no caries experience (DMFT and/or dmft $=0$ ). Higher caries experience was detected in participants that were not born in Hong Kong, had completed active chemotherapy, participated in school dental care service and whose parents had low educational levels. There were 41 patients with active chemotherapy, 24 of whom were diagnosed with acute leukaemia, 5 with haematological malignancies other than leukaemia and 11 with solid tumours. Antimetabolites, cytotoxic antibiotics, alkylating agents and plant alkaloids were administered in $49 \%, 32 \%, 24 \%$ and $22 \%$ of them, respectively. Twenty-six (63\%) patients showed no mucosal complications. The most common oral complication was oral mucositis $(24 \%)$ followed by petechiae $(10 \%)$.

Conclusion: About two-thirds of paediatric and adolescent cancer patients had caries experience, which was more common among those who had completed chemotherapy. Oral mucositis followed by petechiae were the two most common complications of receiving chemotherapy.

Keywords: Caries, mucositis, children, adolescence, Chinese, chemotherapy, cancer.

\section{INTRODUCTION}

In Hong Kong, cancer is the most common diseaserelated cause of death in children [1]. The five most common cancers in children and adolescents up to 19 years old are: leukaemia (27\%), germ-cell and gonadal tumours (15\%), brain and spinal tumours (14\%), carcinoma and epithelial tumours (10\%) and lymphomas (10\%) [2]. The distribution is comparable to many industrialised societies [3-5]. There were about 873,400 children under the age of 15 in Hong Kong with 165 new cases of childhood cancer developed, resulting in 32 new deaths in 2009 [6,7]. Though the figures seem to be painting a rather grim picture, advances in medical science have continued to improve the overall survival of many children with cancer. The overall cure rate of paediatric cancer has increased from less than 50\% in 1970 to almost $80 \%$ at the present day [8]. Oral complications are well recognised sequalae of childhood cancer therapy, with risks of septicaemia, nutritional compromise, significant pain and

*Address correspondence to this author at the Faculty of Dentistry, The University of Hong Kong, 3B61, Prince Philip Dental Hospital, 34 Hospital Road, Hong Kong SAR, China; Tel: 8522859 0287; Fax: 8522858 2532; E-mail: chchu@hku.hk long-term morbidity [9]. By shedding light on these common adverse effects, many cancer studies have aimed to reduce the frequency of oral complications and their impact on disease remission, and to improve the quality of life.

Oral mucositis $(\mathrm{OM})$ is an iatrogenic condition of erythematous inflammatory changes that tend to occur on the buccal and labial surfaces, the ventral surface of the tongue, the floor of the mouth and the soft palate of patients receiving cancer therapy [9]. Its severity ranges from localised or generalised erythema to frank ulceration and haemorrhage [10]. In more severe cases OM can compromise the airway, leading to anoxia-induced brain injury and even death [11]. OM usually starts 7-14 days after the initiation of chemotherapy. Incidence of $\mathrm{OM}$ in children undergoing cancer therapy varies greatly from $10 \%$ to $100 \%$; the apparent diverse range has been suggested to be the result of the lack of a universally agreed instrument in the assessment of mucositis in children [10].

Over the course of cancer therapy, opportunistic infections in the oral cavity such as candidiasis and herpes simplex are probable sequalae as a result of myelosuppression [10]. These infections can be fungal, viral or bacterial infections. Fungal infections often appear with the use of 
broad-spectrum antibiotics and steroids, xerostomia, poor oral hygiene and poor nutrition [12]. Herpes simplex virus (HSV) can cause viral infection, which usually starts out as vesicular lesions periorally or intraorally. The patients often present with pain and distress, drooling and difficulty in swallowing [13]. Bacterial infection is common and not limited to the gingivae. Febrile episodes and systemic bacteraemia may result if the infection is not treated promptly [10].

Graft versus host disease (GVHD) is a major complication associated with haematopoietic stem cell transplantation (HSCT), with clinically significant manifestations in up to half of patients. It frequently leads to poor long-term outcomes and quality of life [10]. It can manifest as acute GVHD or chronic GVHD [14]. Acute GVHD occurs within weeks (about 100 days) of the transplantation procedure. Typical features include fever, diarrhoea, wasting, dermatitis, hepatitis and enteritis [15]. Common oral manifestations range from pain, mucosal erythema, atrophy and ulceration initially to hyperkeratotic and lichenoid changes as the disease progresses [16]. Chronic GVHD usually occurs after day 100 of the transplantation procedure and may directly follow the acute stage or after its resolution, or may develop by itself [15]. It is characterised by lichenoid or scleroderma changes of the skin, keratoconjunctivitis, abnormal liver function, pulmonary insufficiency and intestinal problems. Oral manifestations may include mucosal atrophy, erythema, lichenoid lesions, perioral fibrosis and xerostomia. A literature search found no study reporting the oral health status of paediatric and adolescent oncology patients with chemotherapy in Hong Kong. The objectives of this study were thus to describe the caries experience and the oral mucosal status of children and adolescents with chemotherapy.

\section{MATERIALS AND METHODOLOGY}

This study was performed between December 2010 and February 2011. Ethics approval was sought from the Institutional Review Board (IRB) of the University of Hong Kong/Hospital Authority Hong Kong West Cluster (UW 10294). All paediatric cancer patients aged 18 or below attending the Children's Centre for Cancer and Blood Disease (CCCBD), Department of Paediatrics and Adolescent Medicine, Queen Mary Hospital (QMH), for chemotherapy were invited to join. An invitation letter was distributed to the parents of the patients when they attended the CCCBD. The aims and procedures of the project were explained to the parents. Written consent was sought before patients were accepted into the study.

The study consisted of a questionnaire and a clinical examination. Information concerning the diagnosis, drug history for chemotherapy and haematological analysis [red blood cell, white blood cell, platelet and polymorphonuclear leucocyte (PMN) counts] were obtained, with consent, from the patients' medical records at Queen Mary Hospital.

\section{QUESTIONNAIRE}

Parents of the patients were asked to complete a closedended questionnaire (Appendix VII). It was designed to study the relationship between the following independent variables and the caries experience as well as the mucosal condition among the children.

1. General information - age, gender and place of birth.

2. Family background information-parental educational level and monthly family income.

3. Oral health knowledge of parents-Twenty-one questions taken from a Hong Kong survey on preschool children [17], which focused on the causes and prevention of dental diseases (dental caries and periodontal disease), were asked in order to assess parental dental knowledge. The total score ranging from 0-21 was categorised into three groups: $0-7,8-14$ and 1521. They represented "poor", "moderate" and "good" dental knowledge of parents, respectively.

4. Dental habits of children-Tooth brushing frequency and use of mouthwash.

5. Previous participation in school dental care service (SDCS).

6. Parental satisfaction with their child's oral condition.

\section{CLINICAL EXAMINATION}

Two examination teams, each consisting of one examiner and one recorder, conducted clinical oral examinations and charted dental caries and oral mucosal status. Training of the two examiners and calibration exercises were conducted on patients attending the Paediatric Reception Clinic in Prince Philip Dental Hospital. This was to standardise the assessment of dental caries and oral mucosal status between the examiners and to achieve intra-examiner and inter-examiner agreements. Children were examined in supine position. WHO/CPI 405 probes and disposable intra-oral LED lighted front-surface dental mirrors (Safe T Brite, A.R. Medicom Inc. (Asia) Ltd., Hong Kong, China) were used for clinical examination. Duplicate examinations were carried out on $10 \%$ of the children in order to assess inter-examiner agreement between the two examiners of the study. At the end of the examination, an examination report documenting the oral hygiene status and the number of teeth with caries detected was given to the parents.

\section{DATA ANALYSIS}

The data was imported into a personal computer with Microsoft Office Excel 2007 and screened for errors after data entry. Data analysis was performed using the software Statistical Package for Social Science (SPSS), version 17.0 (SPSS Inc., Chicago, IL, USA). Since a limited sample size was anticipated, the statistical significance was assessed by non-parametric tests (Chi square test or Fisher exact test when the expected values in any of the cells of a contingency table are below 5) of the association between caries experience and gender, place of birth, parental (paternal and maternal) educational level, monthly family income, oral health knowledge of parents, tooth-brushing frequency, use of mouthwash, previous participation in SDCS and parental 
satisfaction with their child's oral condition. Because there are multiple comparisons of the same dataset (caries experience), Bonferroni adjustment was applied and the $\mathrm{p}$ value was set at $0.005(0.05 / 10)$. The inter-examiner reproducibility was assessed with Cohen's Kappa statistic.

\section{RESULTS}

A total of 69 children and adolescent oncology patients were invited, and they all participated in this study. Their mean age was $9.2($ S.D. $=5.0)$ and $44(64 \%)$ were males (Table 1). The Kappa coefficient for inter-examiner agreement of primary and permanent tooth statuses were 0.96 and 0.87 , respectively. Among these 69 participants, 43 (62\%) of them had caries experience (dmft or DMFT). Higher caries prevalence was detected in participants that participated in SDCS (Table 1). The caries experience of primary (dmft) and permanent teeth (DMFT) and the variables studied are shown in Table 2 and Table 3. There was no association found between caries experience of primary teeth (dmft) and the variables studied (Table 2), but a higher caries experience of permanent teeth (DMFT) was found in the participants who were not born in Hong Kong (Table 3).

There were 45 patients aged 12 or below (mean age $6.1 \pm 3.3$ ) with primary or mixed dentition. Their caries experience in mean dmft score was 1.36 \pm 2.21 . Twenty-seven $(60 \%)$ of them had no caries experience $(\mathrm{dmft}=0)$ in their primary teeth, but one child had the highest dmft score of 9 . Seven children $(16 \%)$ had a low caries experience $(0<\mathrm{dmft}$ $\leq 2)$ and $11(24 \%)$ had high caries experience $(\mathrm{dmft}>2)$. Boys were found to have more caries experience (Table 2).

The mean DMFT score of cancer children and adolescents, aged between 6 and 18, undergoing chemotherapy was $2.87 \pm 3.17$. Sixteen $(34 \%)$ children had no caries experience $($ DMFT $=0)$, but the highest DMFT score was 14. Eight children $(17 \%)$ had a low caries experience $(0<\mathrm{dmft} \leq 2)$ and $23(49 \%)$ had high caries experience $(\mathrm{dmft}>2)$. Higher caries experience was found in participants who were not born in Hong Kong or whose mothers had a low educational level (Table 3).

Among the 69 patients, 17 (25\%) were found to present with oral mucosal pathology which included oral mucositis $(\mathrm{N}=12,17 \%)$, petechiae $(\mathrm{N}=4,6 \%)$ and aphthous ulcer $(\mathrm{N}=3$, $4 \%)$. For those with oral mucosal pathology, 15 (88\%) were those under active chemotherapy. The prevalence of oral mucosal pathology among patients with and without active chemotherapy were $37 \%(15 / 41)$ and $7 \%$ (2/28), respectively $(\mathrm{p}=0.009)$. Oral mucosal pathology prevalence according to blood cell count and use of anti-cancer drugs in patients with active chemotherapy is shown in Table $\mathbf{4}$ and Table 5, respectively. Lower white blood cell count and the use of cytotoxic antibiotics and alkylating agents on the patients with active chemotherapy were associated with higher oral mucosal pathology occurrence. Oral mucositis $(\mathrm{N}=10,24 \%)$ was the most common oral mucosal pathology found in the 41 patients with active chemotherapy, followed by petechiae $(\mathrm{N}=4,10 \%)$. Again, lower white blood cell count (Table 4) and the use of cytotoxic antibiotics and alkylating agents (Table 5) were associated with higher oral mucositis occurrence.

\section{DISCUSSION}

This study is a cross-sectional pilot study. The mucosal status at the time of examination might not represent the full effects of chemotherapeutic intervention on the oral mucosa because the presentation of OM varies over the course of chemotherapy. Longitudinal study is more desirable so that patients can be followed up, in particular, with the complications of chemotherapy. This could render a comprehensive correlation between chemotherapy and its adverse effects on oral health. This study recruited patients from QMH, and all invited joined this study. However, the sample size is relatively small for statistical analysis. There are three other hospitals admitting paediatric patients for chemotherapy, which are Tuen Mun Hospital, Prince of Wales and Princess Margaret Hospital. More paediatric cancer patients could be recruited if these hospitals could be included in the study. Oral assessment and follow up of the patients could help better evaluate the relationship between chemotherapy and oral conditions.

Compared to the general population of the children in Hong Kong, the participants in this study had similar caries prevalence in primary teeth [18] and in permanent teeth [19]. It is common to find caries that are left untreated in children [18]. If not arrested, advanced caries can progress into the tooth pulp and eventually form a dental abscess [20], leading to tooth loss and altered dentition. Poor dentition significantly affects children's nutrition and consequently their growth, development and general health. Evidences in current literature between chemotherapy and caries are conflicting and inconclusive [21]. Adverse effects consequent to cancer therapy, particularly salivary gland dysfunction, are known risk factors for dental caries [21]. In our study, prevalence of dental caries in both the primary and permanent dentition was found to be similar to that of the general population. This could be explained by the fact that salivary gland dysfunction induced by chemotherapy is only transient. Similar findings were also made by Dens et al. in long-term, event-free children who had completed cytotoxic treatment: It had no long-term effects on salivary caries risks [22]. In addition, lower caries prevalence was observed in children born in Hong Kong. Water fluoridation, good availability of fluoride toothpaste and easy access to dental care as well as SDCS for children in Hong Kong had contributed to the lower caries experience in children born in Hong Kong [23]. It had previously been shown that low parental educational level was a risk factor of caries in children [17]. In majority of the patients, mothers were the primary caretakers of the children. Perhaps it was the increased dental awareness and knowledge about caries among mothers who had attained secondary education or higher that contributed to the lower caries experience in their children.

Oral mucositis was seen in $25 \%$ of the active patients, much lower than the $60 \%$ incidence reported in the literature [24]. This may be attributed to a variety of factors, including differences in chemotherapy protocols, use of other drugs that may cause $\mathrm{OM}$ with or without concurrent chemotherapy, individual patients' response and the use of agents that may reduce incidence of OM, such as chlorhexidine mouth rinse, on patients. Further investigations are required to verify and account for the differences. This study found more 
Table 1. Caries prevalence of all participants and variables.

\begin{tabular}{|c|c|c|c|}
\hline Variables & Group (N) & Presence $(\%)$ & p-value \\
\hline $\begin{array}{l}\text { Gender } \\
(\mathrm{N}=69)\end{array}$ & $\begin{array}{c}\text { Male (44) } \\
\text { Female (25) }\end{array}$ & $\begin{array}{l}28(64 \%) \\
15(60 \%)\end{array}$ & 0.800 \\
\hline $\begin{array}{l}\text { Diagnosis } \\
(\mathrm{N}=69)\end{array}$ & $\begin{array}{c}\text { Leukaemia (39) } \\
\text { Solid Tumour (19) } \\
\text { Other tumour (11) }\end{array}$ & $\begin{array}{l}23(59 \%) \\
12(63 \%) \\
8(73 \%)\end{array}$ & 0.774 \\
\hline $\begin{array}{l}\text { Chemotherapy } \\
\qquad(\mathrm{N}=69)\end{array}$ & $\begin{array}{c}\text { Active (41) } \\
\text { Completed (28) }\end{array}$ & $\begin{array}{l}22(54 \%) \\
21(75 \%)\end{array}$ & 0.042 \\
\hline $\begin{array}{l}\text { Tooth Brushing Frequency } \\
\qquad(\mathrm{N}=69)\end{array}$ & $\begin{array}{l}\geq \text { Twice daily (46) } \\
<\text { Twice daily (23) }\end{array}$ & $\begin{array}{l}28(61 \%) \\
15(65 \%)\end{array}$ & 0.796 \\
\hline $\begin{array}{l}\text { Use of Mouthwash } \\
\qquad(\mathrm{N}=65)\end{array}$ & $\begin{array}{l}\text { Yes (11) } \\
\text { No (54) }\end{array}$ & $\begin{array}{l}6(55 \%) \\
34(63 \%)\end{array}$ & 0.737 \\
\hline $\begin{array}{l}\text { Joined School Dental Care Service } \\
\qquad(\mathrm{N}=68)\end{array}$ & $\begin{array}{l}\text { Yes (38) } \\
\text { No (30) }\end{array}$ & $\begin{array}{l}29(76 \%) \\
13(43 \%)\end{array}$ & 0.002 \\
\hline $\begin{array}{l}\text { Parental Dental Knowledge } \\
\qquad(\mathrm{N}=69)\end{array}$ & $\begin{array}{c}\text { Good (25) } \\
\text { Moderate (40) } \\
\text { Poor (4) }\end{array}$ & $\begin{array}{l}17(68 \%) \\
23(58 \%) \\
3(75 \%)\end{array}$ & 0.600 \\
\hline $\begin{array}{l}\text { Paternal Educational Level } \\
\qquad(\mathrm{N}=69)\end{array}$ & $\begin{array}{l}<\text { Secondary (11) } \\
\geq \text { Secondary }(58)\end{array}$ & $\begin{array}{l}10(91 \%) \\
33(57 \%)\end{array}$ & 0.043 \\
\hline $\begin{array}{l}\text { Maternal Educational Level } \\
\qquad(\mathrm{N}=69)\end{array}$ & $\begin{array}{l}<\text { Secondary }(7) \\
\geq \text { Secondary }(62)\end{array}$ & $\begin{array}{l}7(100 \%) \\
36(58 \%)\end{array}$ & 0.040 \\
\hline $\begin{array}{l}\text { Monthly Family Income in HK\$ } \\
\qquad(\mathrm{N}=65)\end{array}$ & $\begin{array}{c}<10,000(21) \\
10,000-29999(22) \\
>30000(22)\end{array}$ & $\begin{array}{l}15(71 \%) \\
15(68 \%) \\
11(50 \%)\end{array}$ & 0.327 \\
\hline
\end{tabular}


Table 1. contd...

\begin{tabular}{|c|c|c|c|}
\hline Variables & Group (N) & Presence (\%) & \multicolumn{2}{|c|}{ p-value } \\
\hline \hline \multicolumn{2}{|c|}{} & \multicolumn{2}{|c|}{$\begin{array}{c}6(67 \%) \\
37(62 \%)\end{array}$} \\
\hline $\begin{array}{c}\text { Parental Dental Satisfaction } \\
(\mathrm{N}=69)\end{array}$ & Not satisfied (60) & 1.000 \\
\hline
\end{tabular}

Table 2. Level of caries experience in primary teeth of participants aged below 13 and variables.

\begin{tabular}{|c|c|c|c|c|c|}
\hline \multirow{2}{*}{ Variables } & \multirow{2}{*}{ Group (N) } & \multicolumn{3}{|c|}{ Level of Caries Experience (\%) * } & \multirow{2}{*}{ p-value } \\
\hline & & No & Low & High & \\
\hline $\begin{array}{l}\text { Gender } \\
(\mathrm{N}=45)\end{array}$ & $\begin{array}{c}\text { Male (31) } \\
\text { Female (14) }\end{array}$ & $\begin{array}{l}16(51 \%) \\
1(79 \%)\end{array}$ & $\begin{array}{l}4(13 \%) \\
3(21 \%)\end{array}$ & $\begin{array}{c}11(36 \%) \\
0(0 \%)\end{array}$ & 0.019 \\
\hline $\begin{array}{l}\text { Place of Birth } \\
\qquad(\mathrm{N}=45)\end{array}$ & $\begin{array}{l}\text { Hong Kong (35) } \\
\text { Non-local (10) }\end{array}$ & $\begin{array}{l}21(60 \%) \\
6(60 \%)\end{array}$ & $\begin{array}{l}5(14 \%) \\
2(20 \%)\end{array}$ & $\begin{array}{l}9(26 \%) \\
2(20 \%)\end{array}$ & 0.893 \\
\hline $\begin{array}{l}\text { Diagnosis } \\
(\mathrm{N}=45)\end{array}$ & $\begin{array}{c}\text { Leukaemia (28) } \\
\text { Solid Tumour (9) } \\
\text { Other tumours (8) }\end{array}$ & $\begin{array}{l}18(64 \%) \\
5(56 \%) \\
4(50 \%)\end{array}$ & $\begin{array}{l}5(18 \%) \\
1(11 \%) \\
1(13 \%)\end{array}$ & $\begin{array}{l}5(18 \%) \\
3(33 \%) \\
3(37 \%)\end{array}$ & 0.763 \\
\hline $\begin{array}{l}\text { Chemotherapy } \\
\qquad(\mathrm{N}=45)\end{array}$ & $\begin{array}{c}\text { Active (29) } \\
\text { Completed (16) }\end{array}$ & $\begin{array}{l}19(66 \%) \\
8(50 \%)\end{array}$ & $\begin{array}{l}5(17 \%) \\
2(13 \%)\end{array}$ & $\begin{array}{l}5(17 \%) \\
6(37 \%)\end{array}$ & 0.346 \\
\hline $\begin{array}{l}\text { Tooth Brushing Frequency } \\
\qquad(\mathrm{N}=45)\end{array}$ & $\begin{array}{l}\geq \text { Twice daily (31) } \\
<\text { Twice daily (14) }\end{array}$ & $\begin{array}{l}18(58 \%) \\
9(65 \%)\end{array}$ & $\begin{array}{l}5(16 \%) \\
2(14 \%)\end{array}$ & $\begin{array}{l}8(26 \%) \\
3(21 \%)\end{array}$ & 0.903 \\
\hline $\begin{array}{l}\text { Use of Mouthwash } \\
\qquad(\mathrm{N}=42)\end{array}$ & $\begin{array}{l}\text { Yes (7) } \\
\text { No (35) }\end{array}$ & $\begin{array}{c}4(57 \%) \\
21(60 \%)\end{array}$ & $\begin{array}{l}0(0 \%) \\
6(17 \%)\end{array}$ & $\begin{array}{l}3(43 \%) \\
8(23 \%)\end{array}$ & 0.523 \\
\hline $\begin{array}{c}\text { Joined School Dental Care } \\
\text { Service }(\mathrm{N}=45)\end{array}$ & $\begin{array}{l}\text { Yes (29) } \\
\text { No (16) }\end{array}$ & $\begin{array}{l}21(72 \%) \\
6(38 \%)\end{array}$ & $\begin{array}{l}3(10 \%) \\
4(25 \%)\end{array}$ & $\begin{array}{l}5(18 \%) \\
6(37 \%)\end{array}$ & 0.062 \\
\hline $\begin{array}{l}\text { Parental Dental Knowledge } \\
\qquad(\mathrm{N}=45)\end{array}$ & $\begin{array}{c}\text { Good (18) } \\
\text { Moderate (25) } \\
\text { Poor (2) }\end{array}$ & $\begin{array}{c}8(44 \%) \\
18(72 \%) \\
1(50 \%)\end{array}$ & $\begin{array}{l}3(17 \%) \\
4(16 \%) \\
0(0 \%)\end{array}$ & $\begin{array}{l}7(39 \%) \\
3(12 \%) \\
1(50 \%)\end{array}$ & 0.180 \\
\hline
\end{tabular}


Table 2. contd...

\begin{tabular}{|c|c|c|c|c|c|}
\hline \multirow{2}{*}{ Variables } & \multirow{2}{*}{ Group $(\mathbf{N})$} & \multicolumn{3}{|c|}{ Level of Caries Experience (\%) * } & \multirow{2}{*}{ p-value } \\
\hline & & No & Low & High & \\
\hline $\begin{array}{l}\text { Paternal Educational Level } \\
\qquad(\mathrm{N}=45)\end{array}$ & $\begin{array}{l}<\text { Secondary (6) } \\
\geq \text { Secondary (39) }\end{array}$ & $\begin{array}{l}3(50 \%) \\
24(62 \%)\end{array}$ & $\begin{array}{l}1(17 \%) \\
6(15 \%)\end{array}$ & $\begin{array}{l}2(33 \%) \\
9(23 \%)\end{array}$ & 0.834 \\
\hline $\begin{array}{l}\text { Maternal Educational Level } \\
\qquad(\mathrm{N}=45)\end{array}$ & $\begin{array}{l}<\text { Secondary (4) } \\
\geq \text { Secondary (41) }\end{array}$ & $\begin{array}{l}2(50 \%) \\
25(61 \%)\end{array}$ & $\begin{array}{l}1(25 \%) \\
6(15 \%)\end{array}$ & $\begin{array}{l}1(25 \%) \\
10(24 \%)\end{array}$ & 0.784 \\
\hline $\begin{array}{l}\text { Monthly Family Income in } \\
\qquad \operatorname{HK} \$(N=44)\end{array}$ & $\begin{array}{c}<10,000(16) \\
10,000-29999(18) \\
>30000(10)\end{array}$ & $\begin{array}{l}10(62 \%) \\
9(50 \%) \\
8(80 \%)\end{array}$ & $\begin{array}{c}3(19 \%) \\
4(22 \%) \\
0(0 \%)\end{array}$ & $\begin{array}{l}3(19 \%) \\
5(28 \%) \\
2(20 \%)\end{array}$ & 0.515 \\
\hline $\begin{array}{l}\text { Parental Dental Satisfaction } \\
\qquad(\mathrm{N}=45)\end{array}$ & $\begin{array}{c}\text { Satisfied (5) } \\
\text { Not Satisfied (40) }\end{array}$ & $\begin{array}{l}2(40 \%) \\
25(63 \%)\end{array}$ & $\begin{array}{l}1(20 \%) \\
6(15 \%)\end{array}$ & $\begin{array}{l}2(40 \%) \\
9(22 \%)\end{array}$ & 0.526 \\
\hline
\end{tabular}

*No caries experience: $\mathrm{dmft}=0$; Low caries experience: $0<\mathrm{dmft} \leq 2$; High caries experience: $\mathrm{dmft}>2$

Table 3. Level of caries experience in permanent teeth of participants aged 6 to 18 and variables.

\begin{tabular}{|c|c|c|c|c|c|}
\hline \multirow{2}{*}{ Variables } & \multirow{2}{*}{ Group (N) } & \multicolumn{3}{|c|}{ Level of caries experience $(\%) *$} & \multirow{2}{*}{ p-value } \\
\hline & & No & Low & High & \\
\hline $\begin{array}{l}\text { Gender } \\
(\mathrm{N}=47)\end{array}$ & $\begin{array}{c}\text { Male (29) } \\
\text { Female (18) }\end{array}$ & $\begin{array}{l}11(38 \%) \\
5(27 \%)\end{array}$ & $\begin{array}{l}5(17 \%) \\
3(17 \%)\end{array}$ & $\begin{array}{l}13(45 \%) \\
10(56 \%)\end{array}$ & 0.851 \\
\hline $\begin{array}{l}\text { Place of Birth } \\
\qquad(\mathrm{N}=46)\end{array}$ & $\begin{array}{l}\text { Hong Kong (34) } \\
\text { Non-local (12) }\end{array}$ & $\begin{array}{c}16(47 \%) \\
0(0 \%)\end{array}$ & $\begin{array}{l}4(12 \%) \\
4(33 \%)\end{array}$ & $\begin{array}{l}14(41 \%) \\
8(67 \%)\end{array}$ & 0.005 \\
\hline $\begin{array}{l}\text { Diagnosis } \\
(\mathrm{N}=47)\end{array}$ & $\begin{array}{c}\text { Leukaemia (28) } \\
\text { Solid Tumour (12) } \\
\text { Other tumours (7) }\end{array}$ & $\begin{array}{l}11(39 \%) \\
4(33 \%) \\
1(14 \%)\end{array}$ & $\begin{array}{l}6(22 \%) \\
1(8 \%) \\
1(14 \%)\end{array}$ & $\begin{array}{l}11(39 \%) \\
7(59 \%) \\
5(72 \%)\end{array}$ & 0.495 \\
\hline $\begin{array}{l}\text { Chemotherapy } \\
\qquad(\mathrm{N}=47)\end{array}$ & $\begin{array}{c}\text { Active (26) } \\
\text { Completed (21) }\end{array}$ & $\begin{array}{l}10(38 \%) \\
6(29 \%)\end{array}$ & $\begin{array}{l}4(15 \%) \\
4(19 \%)\end{array}$ & $\begin{array}{l}12(47 \%) \\
11(52 \%)\end{array}$ & 0.792 \\
\hline $\begin{array}{l}\text { Tooth Brushing Frequency } \\
\qquad(\mathrm{N}=47)\end{array}$ & $\begin{array}{l}\geq \text { Twice daily (7) } \\
<\text { Twice daily (37) }\end{array}$ & $\begin{array}{l}12(36 \%) \\
4(29 \%)\end{array}$ & $\begin{array}{l}6(18 \%) \\
2(14 \%)\end{array}$ & $\begin{array}{l}15(46 \%) \\
8(57 \%)\end{array}$ & 0.764 \\
\hline
\end{tabular}


Table 3. contd...

\begin{tabular}{|c|c|c|c|c|c|}
\hline \multirow{2}{*}{ Variables } & \multirow{2}{*}{ Group (N) } & \multicolumn{3}{|c|}{ Level of caries experience $(\%) *$} & \multirow{2}{*}{ p-value } \\
\hline & & No & Low & High & \\
\hline $\begin{array}{l}\text { Use of Mouthwash } \\
\qquad(\mathrm{N}=44)\end{array}$ & $\begin{array}{l}\text { Yes (7) } \\
\text { No (37) }\end{array}$ & $\begin{array}{l}3(43 \%) \\
13(35 \%)\end{array}$ & $\begin{array}{c}0(0 \%) \\
5(14 \%)\end{array}$ & $\begin{array}{l}4(57 \%) \\
19(51 \%)\end{array}$ & 0.583 \\
\hline $\begin{array}{c}\text { Joined School Dental Care } \\
\text { Service }(\mathrm{N}=46)\end{array}$ & $\begin{array}{l}\text { Yes (36) } \\
\text { No (10) }\end{array}$ & $\begin{array}{l}11(31 \%) \\
5(50 \%)\end{array}$ & $\begin{array}{l}7(19 \%) \\
1(10 \%)\end{array}$ & $\begin{array}{l}18(50 \%) \\
4(40 \%)\end{array}$ & 0.568 \\
\hline $\begin{array}{l}\text { Parental Dental Knowledge } \\
\qquad(\mathrm{N}=47)\end{array}$ & $\begin{array}{c}\text { Good (17) } \\
\text { Moderate (27) } \\
\text { Poor (3) }\end{array}$ & $\begin{array}{l}6(35 \%) \\
9(33 \%) \\
1(33 \%)\end{array}$ & $\begin{array}{l}2(12 \%) \\
6(22 \%) \\
0(0 \%)\end{array}$ & $\begin{array}{l}9(53 \%) \\
12(45 \%) \\
2(67 \%)\end{array}$ & 0.930 \\
\hline $\begin{array}{l}\text { Paternal Educational Level } \\
\qquad(\mathrm{N}=47)\end{array}$ & $\begin{array}{l}<\text { Secondary (9) } \\
\geq \text { Secondary (38) }\end{array}$ & $\begin{array}{l}1(11 \%) \\
15(40 \%)\end{array}$ & $\begin{array}{l}2(22 \%) \\
6(15 \%)\end{array}$ & $\begin{array}{c}6(67 \%) \\
17(45 \%)\end{array}$ & 0.256 \\
\hline $\begin{array}{l}\text { Maternal Educational Level } \\
\qquad(\mathrm{N}=47)\end{array}$ & $\begin{array}{l}<\text { Secondary (6) } \\
\geq \text { Secondary (41) }\end{array}$ & $\begin{array}{c}0(0 \%) \\
16(39 \%)\end{array}$ & $\begin{array}{l}3(50 \%) \\
5(12 \%)\end{array}$ & $\begin{array}{l}3(50 \%) \\
20(49 \%)\end{array}$ & 0.025 \\
\hline $\begin{array}{l}\text { Monthly Family Income in } \\
\qquad \operatorname{HK} \$(\mathrm{~N}=43)\end{array}$ & $\begin{array}{c}<10,000(13) \\
10,000-29999(16) \\
>30000(14)\end{array}$ & $\begin{array}{l}2(15 \%) \\
5(31 \%) \\
7(50 \%)\end{array}$ & $\begin{array}{l}4(31 \%) \\
2(13 \%) \\
2(14 \%)\end{array}$ & $\begin{array}{l}7(54 \%) \\
9(56 \%) \\
5(36 \%)\end{array}$ & 0.316 \\
\hline $\begin{array}{l}\text { Parental Dental Satisfaction } \\
\qquad(\mathrm{N}=46)\end{array}$ & $\begin{array}{c}\text { Satisfied (6) } \\
\text { Not Satisfied (40) }\end{array}$ & $\begin{array}{l}2(33 \%) \\
13(34 \%)\end{array}$ & $\begin{array}{l}1(17 \%) \\
7(17 \%)\end{array}$ & $\begin{array}{l}3(50 \%) \\
20(49 \%)\end{array}$ & 1.000 \\
\hline
\end{tabular}

* No caries experience: DMFT $=0$; Low caries experience: $0<\mathrm{DMFT} \leq 2$; High caries experience: DMFT $>2$

Table 4. Prevalence of oral mucosal pathology and oral mucositis according to blood cell count in patients with active chemotherapy $(\mathbf{N}=41)$.

\begin{tabular}{|c|c|c|c|c|c|}
\hline Cell Type & Group $(\mathbf{N})$ & $\begin{array}{c}\text { Prevalence of Oral Mucosal } \\
\text { Pathology (N) }\end{array}$ & p-value & Prevalence of Oral Mucositis (N) & p-value \\
\hline $\mathrm{RBC}$ & $\begin{array}{c}\text { Normal (22) } \\
\text { Abnormal (19) }\end{array}$ & $\begin{array}{l}23 \%(5) \\
53 \%(10)\end{array}$ & 0.600 & $\begin{array}{l}14 \%(3) \\
37 \%(7)\end{array}$ & 0.144 \\
\hline WBC & $\begin{array}{c}\text { Normal (21) } \\
\text { Abnormal (20) }\end{array}$ & $\begin{array}{c}9 \%(2) \\
65 \%(13)\end{array}$ & $<0.001$ & $\begin{array}{l}9 \%(2) \\
40 \%(8)\end{array}$ & 0.032 \\
\hline
\end{tabular}


Table 4. contd...

\begin{tabular}{|c|c|c|c|c|c|}
\hline Cell Type & Group $(\mathbf{N})$ & $\begin{array}{c}\text { Prevalence of Oral Mucosal } \\
\text { Pathology (N) }\end{array}$ & p-value & Prevalence of Oral Mucositis (N) & p-value \\
\hline Platelet & $\begin{array}{c}\text { Normal (23) } \\
\text { Abnormal (18) }\end{array}$ & $\begin{array}{l}26 \%(6) \\
50 \%(9)\end{array}$ & 0.191 & $\begin{array}{l}26 \%(6) \\
22 \%(4)\end{array}$ & 1.000 \\
\hline PMN & $\begin{array}{c}\text { Normal (26) } \\
\text { Abnormal (15) }\end{array}$ & $\begin{array}{l}27 \%(7) \\
53 \%(8)\end{array}$ & 0.108 & $\begin{array}{l}19 \%(5) \\
33 \%(5)\end{array}$ & 0.453 \\
\hline
\end{tabular}

RBC: Normal: $>4 \times 10^{\wedge} 12$; Abnormal: $\leq 4 \times 10^{\wedge} 12$

WBC: Normal: $>4 \times 10^{\wedge} 9$; Abnormal: $\leq 4 \times 10^{\wedge} 9$

Platelet: Normal: $>200 \times 10^{\wedge} 9 ;$ Abnormal: $\leq 200 \times 10^{\wedge} 9$

PMN: Normal: >1x10^9; Abnormal: $\leq 1 \times 10^{\wedge} 9$

Table 5. Use of anti-cancer drugs and prevalence of oral mucosal pathology and oral mucositis ( $N=41)$.

\begin{tabular}{|c|c|c|c|c|c|}
\hline Anti-Cancer Drug & $\begin{array}{l}\text { Use of the Anti- } \\
\text { Cancer Drug (N) }\end{array}$ & $\begin{array}{c}\text { Presence of Mucosal } \\
\text { Pathology }(\%)\end{array}$ & p-value & $\begin{array}{l}\text { Presence of Oral } \\
\text { Mucositis (\%) }\end{array}$ & p-value \\
\hline Antimetabolites & $\begin{array}{l}\text { Yes (20) } \\
\text { No (21) }\end{array}$ & $\begin{array}{l}9(45 \%) \\
6(29 \%)\end{array}$ & 0.341 & $\begin{array}{l}6(30 \%) \\
4(19 \%)\end{array}$ & 0.484 \\
\hline Cytotoxic antibiotics & $\begin{array}{l}\text { Yes (13) } \\
\text { No (28) }\end{array}$ & $\begin{array}{l}10(77 \%) \\
5(18 \%)\end{array}$ & $<0.001$ & $\begin{array}{l}7(54 \%) \\
3(11 \%)\end{array}$ & 0.005 \\
\hline Alkylating agent & $\begin{array}{l}\text { Yes (10) } \\
\text { No (31) }\end{array}$ & $\begin{array}{l}6(60 \%) \\
9(29 \%)\end{array}$ & 0.013 & $\begin{array}{l}5(50 \%) \\
5(16 \%)\end{array}$ & 0.045 \\
\hline Plant alkaloids & $\begin{array}{l}\text { Yes (9) } \\
\text { No (32) }\end{array}$ & $\begin{array}{l}4(44 \%) \\
11(34 \%)\end{array}$ & 0.701 & $\begin{array}{l}3(33 \%) \\
7(22 \%)\end{array}$ & 0.662 \\
\hline $\begin{array}{c}\text { Topoisomerases } \\
\text { inhibitor }\end{array}$ & $\begin{array}{l}\text { Yes (13) } \\
\text { No }(28)\end{array}$ & $\begin{array}{l}7(54 \%) \\
8(29 \%)\end{array}$ & 0.168 & $\begin{array}{l}4(31 \%) \\
6(21 \%)\end{array}$ & 0.698 \\
\hline RTK inhibitor & $\begin{array}{l}\text { Yes (5) } \\
\text { No (36) }\end{array}$ & $\begin{array}{l}2(40 \%) \\
13(36 \%)\end{array}$ & 1.000 & $\begin{array}{l}1(20 \%) \\
9(25 \%)\end{array}$ & 1.000 \\
\hline
\end{tabular}

$\mathrm{OM}$ in patients taking cytotoxic antibiotics and alkylating agents, which is in agreement with that reported in other studies [25]. Cytotoxic antibiotics interfere with DNA/RNA synthesis in cells, while alkylating agents attach an alkyl group to DNA in body cells. Oral mucosal epithelial cell renewal is thus affected, leading to atrophy and ulceration
[26]. Leukopenia had been found to be associated with the occurrence of OM; this is also consistent with the findings by Raber-Durlacher et al. [27]. Sonis suggested that neutropenia predisposed ulcerated oral mucosa to superinfection that aggravated the mucositis [26]. Meanwhile, no significant relationship has been found between OM and counts of other blood cell types. 
Haematopoietic stem cell transplantation (HSCT) offers a possible cure to many haematological malignancies [28]. However, GVHD may arise as a result of allogenic donor cells' response to host tissues. Oral lesions, ranging from hyperkeratosis to ulceration, are commonly seen [29]. Treatment using immunosuppressants and corticosteroids predisposes patients to opportunistic infection. Also, cyclosporine, the most widely used immunosuppressant, is a well-known cause of drug-induced gingival hyperplasia.

Among the parents interviewed in the study, although around one-third of them had good dental knowledge, the majority had quite a number of misconceptions about the causes of tooth decay, the effects of fluoride on tooth substance and the causes of periodontal disease and its prevention. Clearly, the level of dental knowledge of the parents is yet to be improved. And since poor oral hygiene was associated with more severe OM [30], oral hygiene education should be reinforced to minimise the effects of chemotherapy on the oral tissues and to maintain oral health.

Patient satisfaction with oral health is considered an important factor to motivate patients to improve oral health [31, 32]. The majority of parents were not satisfied with their children's oral health conditions. One would naturally assume that parents who are not satisfied with their children's oral health would pay more attention to it, either through vigorous oral hygiene practice or professional dental treatment, which should reduce caries incidence. However, parental satisfaction was not found to be associated with the patients' caries experience in this study. Perhaps this was because with cancer therapy on their mind oral health was not their priority. On the other hand, their dissatisfaction with oral conditions might be related to painful mucosal changes rather than caries.

\section{CONCLUSION}

In this pilot study, the majority of the children and adolescents with chemotherapy in Hong Kong had caries experience. The caries experience of primary teeth in children aged 12 or below with cancer therapy measured by mean $\mathrm{dmft}$ index was 1.36, and the caries experience of boys was higher than that of girls. The caries experience of permanent teeth of patients aged 6 to 18 with chemotherapy measured by mean DMFT index was 2.8,; and those who were not born in Hong Kong and with low maternal educational level had higher caries experience. Most of the oral mucosal pathologies were found in patients under active chemotherapy; and oral mucositis followed by petechiae were the two most common complications.

\section{TRIAL REGISTRATION}

This study has been registered with the HKU Clinical Trial Register and is available for public access on the Internet (www.HKClinicalTrials.com). The study identifier is: HKCTR-1119 Title: Community dental care for children undergoing cancer therapy. Ethics approval was sought from the Institutional Review Board (IRB) of the University of Hong Kong/ Hospital Authority Hong Kong West Cluster (UW 10-294).

\section{CONFLICT OF INTEREST}

The authors confirm that this article content has no conflicts of interest.

\section{ACKNOWLEDGEMENTS}

This study was financially supported by the Faculty of Dentistry. The authors wish to thank the children and adolescents, as well as their families, who joined the study. The authors also thank the staff at the ward and outpatient clinic of the Department of Paediatrics and Adolescent Medicine, Queen Mary Hospital, for patient recruitment. This study has been developed as a community health project, and the authors would like to thank Drs. CK Cheung, SL Cheung, MN Kwok, WS Yuen, KT Tang, ML Tsang and SW Wong for their contributions to the study.

\section{REFERENCES}

[1] Lau YL. Health Indicators. HK J Paediatr 1999; 4: 52-4.

[2] Hong Kong Cancer Registry, Hospital Authority. Hong Kong Cancer statistics 2007; [cited 2014 June 6]. Available from: http://www3.ha.org.hk/cancereg/e_canstat2007.pdf

[3] National Cancer Institute. Cancer Incidence and Survival Among Children and Adolescents: United States SEER Program 19751995; [cited 2014 June 6]. Available from: http://www.seer.cancer.gov/publications/childhood-monograph.pdf/

[4] Office of National Statistics. Childhood cancer, Great Britain (percentages), Office for national statistics, Great Britain; [cited 2014 June 6]. Available from: http://www.statistics.gov.uk/cci/nugget.asp?id=681

[5] Baade PD, Youlden DR, Valery PC, et al. Trends in incidence of childhood cancer in Australia, 1983-2006. Br J Cancer 2010; 102(3): 620-6.

[6] Information Services Department, Hong Kong Special Administrative Region Government. Hong Kong: The Facts - Population; [cited 2014 June 6]. Available from: http://www.gov.hk/en/about/abouthk/factsheets/docs/population.pdf

[7] Children's Cancer Foundation; [cited 2014 June 6]. Available from: http://www.ccf.org.hk/

[8] Raab CP, Gartner JC. Diagnosis of Childhood Cancer. Prim Care Clin Office Pract 2009; 36(4): 671-84.

[9] Simon AR, Roberts MW. Management of oral complications associated with cancer therapy in pediatric patients. J Dent Child 1991; 58 (5): 384-9.

[10] Hong $\mathrm{CH}$, daFonseca M. Considerations in the Pediatric Population with Cancer. Dent Clin North Am 2008; 52(1): 155-81.

[11] Sonis ST. Mucositis as a biological process: a new hypothesis for the development of chemotherapy - induced stomatotoxicity. Oral Oncol 1998; 34(1): 39-43.

[12] Glick M, Siegel MA. Viral and fungal infections of the oral cavity in immunocompetent patients. Infect Dis Clin North Am 1999; 13(4): 817-31.

[13] Woo SB, Sonis ST, Sonis AL. The role of herpes simplex virus in the development of oral mucositis in bone marrow transplant recipients. Cancer 1990; 66(11): 2375-9.

[14] Woo SB, Lee SJ, Schubert MM. Graft-vs.-host disease. Crit Rev Oral Biol Med 1997; 8(2): 201-16.

[15] Horwitz ME, Sullivan KM. Chronic graft-versus-host disease. Blood Rev 2006; 20(1): 15-27.

[16] Chu $\mathrm{CH}$, Lee $\mathrm{AH}$, Zheng L, et al. Arresting rampant dental caries with silver diamine fluoride in a young teenager suffering from chronic oral graft versus host disease post-bone marrow transplantation: a case report. BMC Res Notes 2014; 7:3.

[17] Chu CH, Lo EC. Patients' satisfaction with dental services provided by a university in Hong Kong. Int Dent J 1999; 49(1): 53-9.

[18] Chu CH, Ho PL, Lo ECM. Oral health status and behaviours of preschool children in Hong Kong. BMC Public Health 2012; 12: 767. 
[19] Zhang SN, Chau AMH, Lo ECM, Chu CH. Dental caries and erosion status of 12-year-old Hong Kong children. BMC Public Health 2014; $14: 7$.

[20] Chu CH. Treatment of early childhood caries: A review and case report. General Dentistry 2000; 48(2): 42-48.

[21] Wogelius P, Dahllöf G, Gorst-Rasmussen A, et al. A populationbased observational study of dental caries among survivors of childhood cancer. Pediatr Blood Cancer 2008; 50(6): 1221-6.

[22] Dens FL, Boute P, Vinckier F, Declerck D. Salivary caries risk factors in long-term event-free survivors of pediatric malignant diseases. J Clin Pediatr Dent 1996; 20(3): 241-5.

[23] Chu CH, Wong SSS, Suen RPC, Lo ECM. Oral health and dental care in Hong Kong. Surgeon 2013; 11(3): 153-7.

[24] Cheng KK, Molassiotis A, Chang AM, Wai WC, Cheung SS. Evaluation of an oral care protocol intervention in the prevention of chemotherapy-induced oral mucositis in paediatric cancer patients. Eur J Cancer 2001; 37(16): 2056-63.

[25] Kostler W, Hejna M, Wenzel C, Zielinski C. Oral Mucositis Complicating Chemotherapy and/or Radiotherapy: Options for Prevention and treatment. CA Cancer J Clin 2001; 51(5): 290-315.
[26] Sonis ST, Elting LS, Keefe D, et al. Perspectives on cancer therapy-induced mucosal injury. pathogenesis, measurement, epidemiology, and consequences for patients. Cancer 2004; 100(9 Suppl): 1995-2025.

[27] Raber-Durlacher JE, Weijl NI, Abu Saris M, de Koning B, Zwinderman AH, Osanto S. Oral mucositis in patients treated with chemotherapy for solid tumors: a retrospective analysis of 150 cases. Support Care Cancer 2000; 8(5): 366-71.

[28] Edward A, Copelan EA. Hematopoietic stem-cell transplantation. N Engl J Med 2006; 354: 1813-26.

[29] Schubert MM, Correa ME. Oral graft-versus-host disease. Dent Clin North Am 2008; 52(1): 79-109.

[30] Wuketich S, Hienz SA, Marosi C. Prevalence of clinically relevant oral mucositis in outpatients receiving myelosuppressive chemotherapy for solid tumors. Support Care Cancer 2012; 20(1):175-83.

[31] Chu CH, Fung DS, Lo EC. Dental caries status of preschool children in Hong Kong. Br Dent J 1999; 187(11): 616-20.

[32] Chu CH, Yeung CY, Lo EC. Monitoring patient satisfaction with university dental services under two fee-paying systems. Community Dent Oral Epidemiol 2001; 29(5): 390-8.

Received: October 01, 2014

Revised: December 08, 2014

Accepted: December 14, 2014

() Kung et al.; Licensee Bentham Open.

This is an open access article licensed under the terms of the Creative Commons Attribution Non-Commercial License (http://creativecommons.org/licenses/by-nc/3.0/) which permits unrestricted, non-commercial use, distribution and reproduction in any medium, provided the work is properly cited. 